\title{
MOLECULAR DYNAMIES CALCULATIONS ON THE TIME DEPENDENCE OF SIMPLE, ANISOTROPIC POTENTIALS IN DENSE ARGON
}

\author{
D. FRENKEL and J. VAN DER ELSKEN \\ Laboratory for Ptiysical Chemistry, University of A msterdam, Amsterdam, The Netherlands
}

Received 9 February 1976

\begin{abstract}
Molecular dynamics calculations on the time dependence of the anisotropic potential experienced by a probe molecule in argon, are presented. The calculations were performed for Ar densities of 100, 300, 500 and 784 amagat and $T=160 \mathrm{~K}$. Collective motions clearly manifest themselves at higher densities, in particular in the power-spectrum of the part of the anisotropic potential that transforms as an irreducible tensor of rank 1. The relation between time-dependent local anisotropy and a number of experiments is discussed.
\end{abstract}

\section{Introduction}

Very little is known about the relaxation of anisotropies in fluids. Possibly because the time dependence of local anisotropy is not connected as directly to any experiment as for instance time-dependent density fluctuations are to neutron- and light-scattering. Time-dependent local anisotropies, however, do play an important role in the rotational relaxation of small probe molecules in a host fluid $[1,2]$. To be more precise, rotational relaxation is due to the time-dependent anisotropic potential that perturbs the probe molecule. We will derive the relation between this perturbation and the local anisotropy in the density of the host fluid around a linear probe molecule.

The interaction between a inear molecule and a single, atomic perturber can be formally expanded in a series of Legendre polynomials:

$$
V(r, \theta)=\sum_{l=0}^{\infty} v_{l}(r) P_{l}(\cos \theta)
$$

where $r$ is the molecule-perturber distance and $\theta$ the angle between the molecular axis and the line joining the centers of mass of molecule and perturber. Using the spherical harmonics addition theorem [3], this may be rewritten as

$$
V(r, \theta, \phi)=\sum_{l=0}^{\infty}[4 \pi /(2 l+1)] v_{l}\left(r_{p}\right) \sum_{m=-l}^{l} Y_{b n}^{*}\left(\theta_{p}, \phi_{p}\right) Y_{l m}(\theta, \phi),
$$

where $\theta_{p}, \phi_{p}$ now fix the orientation of the vector $r_{p}$ with respect to a fixed frame and $\theta, \phi$ describe the orientation of the molecule in the same frame. If $N$ perturbers are present, the perturbing potential may be written as:

$$
V(\theta, \phi)=\sum_{l=0}^{\infty}[4 \pi /(2 l+1)] \sum_{p=1, N} v_{l}\left(r_{p}\right) \sum_{m=-l}^{l} Y_{l m}^{*}\left(\theta_{p}, \phi_{p}\right) Y_{l m}(\theta, \phi) .
$$

Here it is assumed that all contributions to the potential are pairwise additive. Introducing the perturber density at time $t, \rho(r, t)=\Sigma_{p=i, N} \delta\left(r-r_{p}(t)\right)$, the above expression can be rewritten as:

$$
V(\theta, \phi ; t)=\sum_{l=0}^{\infty}[4 \pi /(2 l+1)] \sum_{m=-l}^{l}\left[\int_{0}^{\infty} \int_{0}^{\pi} \int_{0}^{2 \pi} \rho\left(r^{\prime}, t\right) v_{l}\left(r^{\prime}\right) Y_{l m}\left(\theta^{\prime}, \phi^{\prime}\right)\left(r^{\prime}\right)^{2} \sin \theta^{\prime} \mathrm{d} r^{\prime} \mathrm{d} \theta^{\prime} \mathrm{d} \phi^{\prime}\right] Y_{b r l}(\theta, \phi)
$$




$$
\equiv \sum_{l=0}^{\infty}[4 \pi /(2 l+1)]: \sum_{m=-l}^{l} a_{l m}(t) Y_{l m}(\theta, \phi)
$$

The coefficients $a_{b m}(t)$ defined above, are determined by the time dependence of that part of the local density around a probe molecule that transforms as $Y_{I m}(\theta, \phi)$.

Any description of the rotational relaxation of a probe molecule requires the knowledge of the time-dependent perturbation. To get some insight into the statistical properties that characterize this time-dependent perturbation, it is profitable to know its power spectrum. The knowledge of the power spectrum gives; first of all, an idea what frequency components of the perturbation are predominantly involved at different densities, and secondly, if a perturbation approach is justified, the knowledge of these frequency components is even sufficient for quantitative results [1]. It is therefore of some practical interest to know the correlation function of the perturbing potential. For a given molecule-perturber potential, all $a_{I m}(t)$, and hence the correlation function, can be calculated by molecular dynamics (MD).

In order for the MD approach to be feasible, one has to assume that the translational motion is classical and that the anisotropic potential does not influence the translational motion. The correlation function of the perturbation is written as:

$$
\left\langle V\left(\theta^{\prime}, \phi^{\prime} ; 0\right) V(\theta, \phi ; t)\right)=\sum_{l, l^{\prime}=0}^{\infty}\left[16 \pi^{2} /(2 l+1)\left(2 l^{\prime}+1\right)\right] \sum_{m=-l m^{\prime}=-l^{\prime}}^{l}\left\langle a_{l^{\prime} m^{\prime}}(0) a_{l m}(t)\right\rangle Y_{l^{\prime} m^{\prime}}\left(\theta^{\prime}, \phi^{\prime}\right) Y_{l m}(\theta, \phi)
$$

Because, on the average, the fluid is isotropic, all $\left.G_{l^{\prime} m^{\prime}}(0) a_{l m}(t)\right)$ are zero, except if $l^{\prime}=l$ and $m^{\prime}=-m$, moreover $(-1)^{m}\left\langle a_{l-m}(0) a_{l m}(t)\right\rangle$ is independent of $m$;

$$
(-1)^{m}\left\langle a_{l-m}(0) a_{l m}(t)\right\rangle=\left\langle a_{l 0}(0) a_{l 0}(t)\right\rangle \equiv\left\langle C_{l}(0) C_{l}(t)\right\rangle(2 i+1) / 4 \pi
$$

where $C_{l}(t)$ is defined by

$$
C_{l}(t) \equiv \int_{0}^{\infty} \int_{0}^{\pi} \int_{0}^{2 \pi} \rho(r, t) v_{l}(r) P_{l}(\cos \theta) r^{2} \sin \theta \mathrm{d} r \mathrm{~d} \theta \mathrm{d} \phi
$$

The correlation function of the perturbing potential can therefore be written as:

$$
\left\langle V\left(\theta^{\prime}, \phi^{\prime} ; 0\right) V(\theta, \phi ; t)\right\rangle=\sum_{l=0}^{\infty}[4 \pi /(2 l+1)]\left\langle C_{l}(0) C_{l}(t)\right\rangle \sum_{m=-I}^{l}(-1)^{m} Y_{l-m}\left(\theta^{\prime}, \phi^{\prime}\right) Y_{l m}(\theta, \phi)
$$

It should be noted that the correlation function is a sum of independent correlation functions for different $l$. We will now concentrate on the time-dependent part of (8). In order to calculate $C_{l}(t)$ one needs to know the $r$-dependence of the anisotropic potential $v_{l}(r)$. In the computations described below $v_{1}(r)$ and $v_{2}(r)$ were assumed to be proportional to the isotropic potential, $v_{0}(r)$. Although it is known that the $r$-dependence of $v_{l}(r)$ may differ for different $l$, one may expect that the general features of $\left\langle C_{l}(0) C_{l}(t)\right)$ are not very dependent on the exact nunierical values chosen for $v_{f}(r)$, as long as the overall shape and range are correct.

\section{Calculations}

Molecular dynamics calculations were performed for a system of 256 Lennard-Jones ("argon") atoms. The time step corresponcied to $10^{-14} \mathrm{~s}$, the temperature to $160 \mathrm{~K}( \pm 1 \%)$. Runs of 5000 time steps were made at densities of $0.106,0.319,0.531$, and 0.833 in units $\rho \sigma^{3}$. This corresponds to argon densities of 100,300,500, and 784 amagat, i.e. extending from dense gas to typical liquid densities. The value of $C_{1}(t)$ and $C_{2}(t)$ was calculated every $2 \times 10^{-14}$ s. The $r$-dependence of the anisotropic potentials $v_{1}(r)$ and $v_{2}(r)$ was assumed to be proportional to $v_{0}(r)=$ $4 \epsilon\left[(\sigma / r)^{12}-(\sigma / r)^{6}\right]$, with $\epsilon=119.5 \mathrm{~K}$ and $\sigma=3.405 \AA$. As all particles in the MD calculations had the same mass, the results of the calculations should be applicable to cases in which the mass of the probe molecule and the host 
atoms is approximately equal. Every particle was successively considered as the probe molecule, thus improving the statistics by a factor 256 . From the data generated in the MD runs, the correlation functions $\left\langle C_{1}(0) C_{1}(r)\right\}$ and $\left\langle C_{2}(0) C_{2}(t)\right\rangle$ were calcuiated; these are shown in figs. 1 and 2 . Figs. 3 and 4 show their Fourier transforms $G_{1}(\omega)$ and $G_{2}(\omega)$.

\section{Discussion}

As can be seen from figs. 1 and 2 , increasing the density has the effect to speed up the decay of the correlation functions of the $l=1$ and $l=2$ arisotropic potentials. At low densities the decay time is typically of the order of $5 \times 10^{-12} \mathrm{~s}$, whereas at the highest density the relaxation is about an order of magnitude faster. Another striking effect to be noted is the behaviour of $G_{1}(\omega)$ and $G_{2}(\omega)$ at high densities. As can be seen in fig. 3, a small hump at $60 \mathrm{~cm}^{-1}$ in the power spectrum of $C_{1}(t)$ at a density of 100 amagat increases rapidly with density to a strong peak at $784 \mathrm{Am}$, almost equal in intensity to the remnant of the zero frequency peak. The corresponding correlation functions (fig. 1), show an evolution from slow, almost monotonic, decay to a rapid oscillatory behaviour at the highest density. In $G_{2}(\omega)$ there is clearly also a large increase in the higher frequency components with density, although a distinct peak does not evolve. It is appealing to ascribe the rapid increase in intensity of the power spectrum of $C_{1}(t)$ around $60 \mathrm{~cm}^{-1}$, to the onset of collective motions in the fluid. Indeed, the maximum of the frequency distribution of liquid argon is situated in precisely this same region. Such an assumption would also qualitatively explain the different behaviour of $G_{1}(\omega)$ and $G_{2}(\omega)$. If a particle, taking part in a phonon-like motion, is initially close to one of its neighbours, it will be closer to the neighbour in the opposite direction, half a period later. Therefore, if the $z$-axis is chosen, for the moment, in the direction of the particle displacement, $C_{1}(t)$ will change sign in half a period whereas $C_{2}(t)$ will not. For this reason the power spectrum of $C_{1}(t)$ resembles the frequency distribution of the medium closer than does the power spectrum of $C_{2}(t)$. Another point that should be noted is

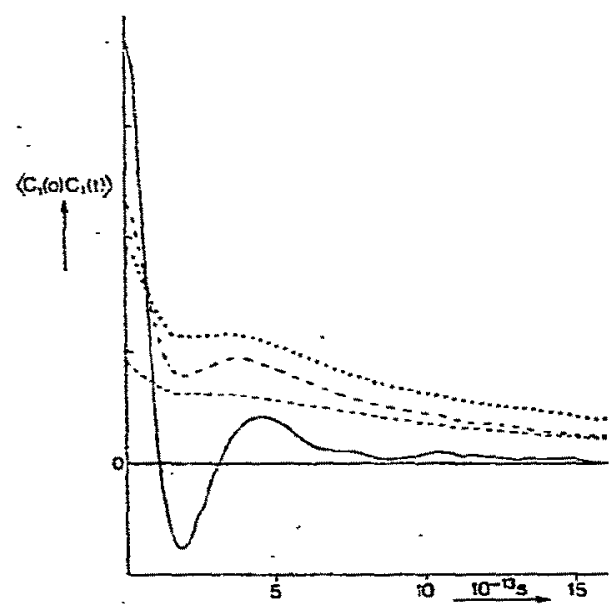

Fig. 1. The correlation function of $C_{2}(t)$, the part of the intermolecular potential that transforms as an irreducible tensor of rank I, at $T=160 \mathrm{~K}$, as a furiction of the Ar dersity. The correlation function at different densities is drawn to the same, arbitrary scale. 100 amagat $(-\cdots)$; 300 amagat $(\cdots) ; 500$ amagat $(-\cdot-\cdot-) ; 784$ amagat $(-)$.

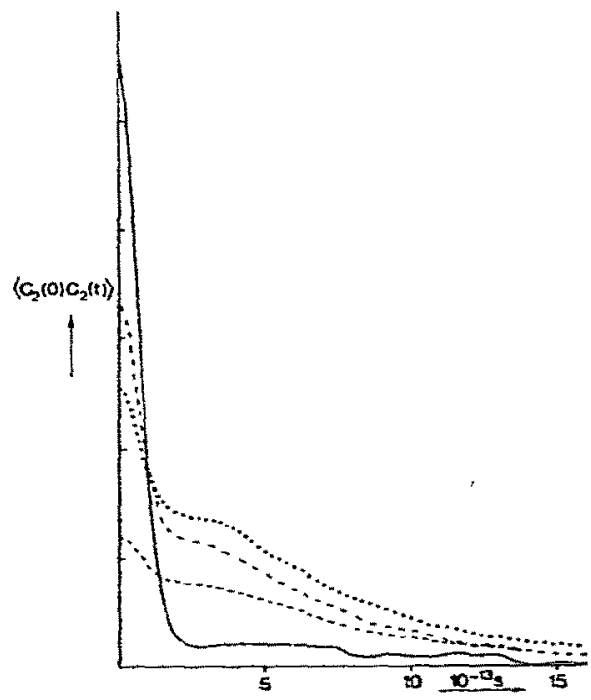

Fig. 2. The correlation function of $C_{2}(t)$, the part of the intermolecular potential that transforms as an irreducible tensor of rank 2 , at $T=160 \mathrm{~K}$, as a function of the Ar density. The correlation function at different densities is drawn to the same, arbitrary scale. 100 amagat $(-\ldots) ; 300$ amagat $(\ldots) ; 500$ anagat (-..-- ); 784 amagat (-). 
that the correlation functions shown in figs. 1 and 2 are, at liquid densities, clearly not exponential. Exponential decay of anisotropic perturbations in liquious has been assumed by several authors $[1,4]$. For argon, such an as: sumption does not seem justified.

Experimental data on time-dependent anisotropies in dense fluids are rather scarce. In the case of argon, relevant information seems to be contained in the following types of experiments. First of all, depolarized collision-induced light scattering $[5,6]$ gives information about the decay of anisotropies of the $P_{2}$ type. This phenomenon has been investigated by Alder et al. [7] in an extensive MD study. Although the description of depolarized collision-induced light scattering is more involved than the description of anisotropic perturbations, as discussed in this letter, because time-dependent four-particle correlations may be involved as opposed to three-particle correlations in our case, it is tempting to compare the results of Alder et al. with the behaviour we observe for $C_{2}(t)$ :

First of all, one should note that, whereas in the above calculations the $P_{2}$ anisotropy in the density is weighted by a $\mathrm{L}$ potential, the corresponding weight function in ref. [7] is the $r^{-3}$ dependence of the dipole-induced dipole contributions to depolarized light-scattering. This implies that the local anisotropy is probed over different ranges. Nevertheless the correlation function $\left\langle C_{2}(0) C_{2}(t)\right\rangle$ is, at liquid densities, qualitatively very similar to the corresponding correlation function (called $\rho(\tau)$ ) of ref. [7].

However, at lower densities the decay of $\rho(\tau)$ seems to be faster than that of $\left\langle C_{2}(0) C_{2}(t)\right\rangle$.

Another experimental technique that is sensitive to the time dependence of local anisotropy is collision-induced dipolar absorption spectroscopy. From these experiments [8] the power spectrum of the dipole, induced by the interaction between the host fluid and a probe atom, is obtained. As the induced dipole moment transforms as an $l=1$ spherical harmonic, these power spectra should be compared with $G_{1}(\omega)$. If the concentration of the probe atoms is low, only three-particle correlations enter in the description of the dipole correlation function $\left\langle\mu_{\text {ind }}(0) \mu_{\text {ind }}(t)\right\rangle$. The essential difference with $C_{1}(t)$ is that the induced dipole moment decays much faster as a function of interparticle distance (in fact, to a very good approximation, exponentially) than the $\mathbf{L}$ potential: Therefore collision-induced dipolar absorption typically probes short range anisotropy. This different range makes

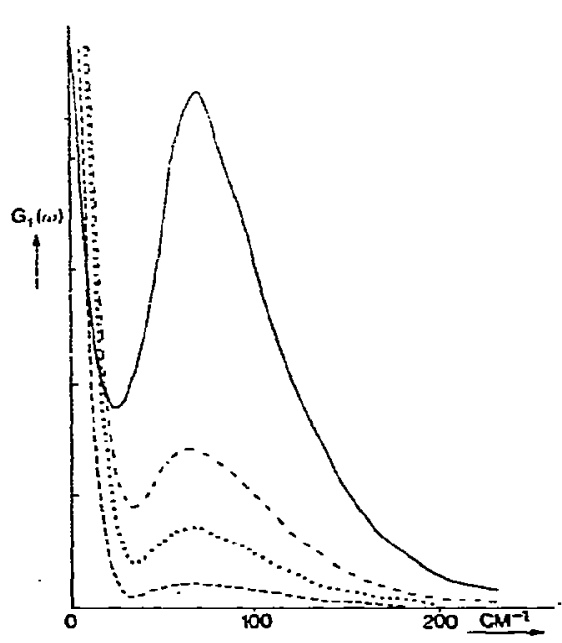

Fig. 3. The power spectrum of $C_{1}(t)$ at $T=160 \mathrm{~K}$, as a function of the Ar density. The power spectrum at different densities is drawn to the same, arbitrary scale. The large, but finite values of $G_{1}(0)$ fall outside the range of the picture, except for the highest density. 100 amagat $(---) ; 300$ amagat $(\cdots)$; 500 amagat (-..--); 784 amagat (-).

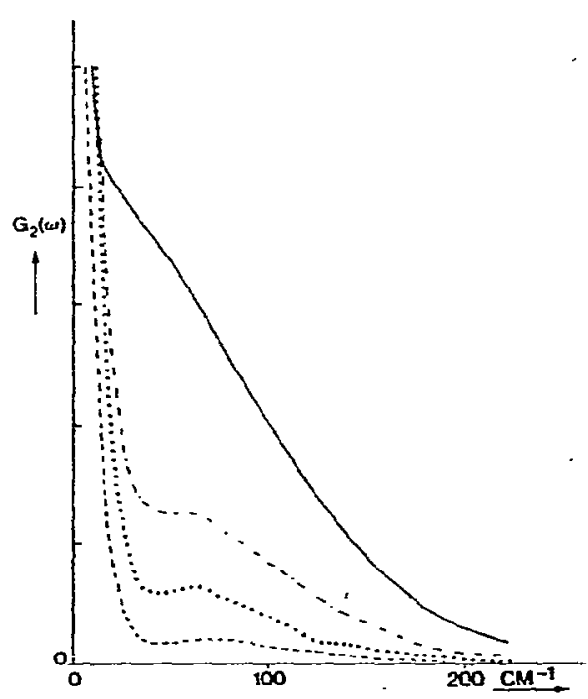

Fig. 4. The power spectrum of $C_{2}(t)$ at $T=160 \mathrm{~K}$, as a function of the Ar density. The power spectrum at different densities is drawn to the same, arbitrary scale. The large, but finite values of $G_{2}(0)$ fall outside the range of the picture. 100 amagat $(--\rightarrow) ; 300$ amagat $(. .) ;$.500 amagat $(-\cdot-\cdot-) ; 784$ amagat $(\longrightarrow)$. 
the power spectrum of $\mu_{\text {ind }}(t)$ look quite different from $G_{1}(\omega)$. The strong coupling of $P_{1}$ anisotropy with collective modes does, however, show up in this case as well. In fact, it manifests itself through the presence of the intercollisional interference dip [9].

The relation beween rotational line-broadening and the power spectrum of the perturbing potential is simple if a perturbation appooach is justified and if the time scale of rotational relaxation is larger than the characteristic time for the dissipation of local anisotropy. Under these conditions, there is a linear relation between rotational line-widths and the power spectrum of the perturbing potential. In rotational relaxation experiments, however, the power spectrum of the perturbing potential is only probed at a number of discrete frequencies, corresponding to possible transitions between the energy levels of the probe molecule, including transitions between degenerate levels.

In this discussion it was implicitly assumed that collision-induced light scattering as well as collision-induced dipolar absorption arise from pairwise additive contributions. The same assumption was made in the first section about the anisotropic potential perturbing a probe molecule. Within the limits of this approximation, it is clear that rotational relaxation measurements can provide important complementary information on the time dependerice of local anisotropy in dense fluids. In particular it enables one to probe a different range of the three-particle correlation function than eicher collision-induced light scattering or collision-induced dipolar absorption.

\section{Acknowledgement}

This work is part of the research program of the Foundation for Fundamental Research of Matter (F.O.M.) and was made possible by financial support from the Netheriands Organisation for Pure Research (Z.W.O.). The calculations described in this letter were performed at CECAM in Orsay. One of the authors (D.F.) wishes to thank Dr. C. Moser of CECAM for the granted hospitality. We gratefully acknowledge fruitful discussions with Dr. J.D. Poll and Dr. G. Jacucci.

\section{References}

[1] D. Robert and L. Galatry, J. Chem. Phys. 55 (1971) 2347.

[2] D. Frenkel, D.J. Gravesteyn and J. van der Elsken, Chem. Phys. Letters 40 (1976) 9.

[3] M.E. Rose, Elementary theory of angular momentum (Viley, New York, 1957) ch. 4.

[4] H. Shimizu, J. Chem. Phys. 43 (1965) 2453.

[5] W.S. Gornall, H.E. Howard-Lock and B.P. Stoicheff, Phys. Rev. A1 (1970) 1288.

[6] P.A. Fleury, W.B. Daniels and J.M. Worlock, Phys. Rev. Letters 27 (1971) 1492.

[7] B.J. Alder, H.L. Strauss and J.J. Weis, J. Chem. Phys. 59 (1973) 1002.

[8] U. Buontempo, S. Curisolo and G. Jacucci, Can. J. Phys. 49 (1971) 2870.

[9] J. van Kranendonk, Can. J. Phys. 46 (1968) 1173. 